\title{
Staphylococcal lipoteichoic acid inhibits delayed-type hypersensitivity reactions via the platelet-activating factor receptor
}

\author{
Qiwei Zhang, ${ }^{1,2}$ Nico Mousdicas, ${ }^{1}$ Qiaofang Yi, ${ }^{1}$ Mohammed Al-Hassani, ${ }^{1}$ \\ Steven D. Billings, ${ }^{1,3}$ Susan M. Perkins, ${ }^{4}$ Katherine M. Howard, ${ }^{5}$ Satoshi Ishii, ${ }^{6}$ \\ Takao Shimizu, ${ }^{6}$ and Jeffrey B. Travers ${ }^{1,2,7,8}$
}

\begin{abstract}
1Department of Dermatology, ${ }^{2}$ Department of Pediatrics and the H.B. Wells Center for Pediatric Research, ${ }^{3}$ Department of Pathology and Laboratory Medicine, and ${ }^{4}$ Division of Biostatistics, Indiana University School of Medicine, Indianapolis, Indiana, USA. ${ }^{5}$ Department of Biochemistry,

University of Texas Health Sciences Center, San Antonio, Texas, USA. ${ }^{6}$ Department of Biochemistry and Molecular Biology, Faculty of Medicine, University of Tokyo, Tokyo, Japan. ${ }^{7}$ Department of Pharmacology and Toxicology and ${ }^{8}$ Richard L. Roudebush V.A. Medical Center, Indiana University School of Medicine, Indianapolis, Indiana, USA.
\end{abstract}

\begin{abstract}
Staphylococcus aureus infections are known triggers for skin inflammation and can modulate immune responses. The present studies used model systems consisting of platelet-activating factor receptor-positive and -negative (PAF-R-positive and -negative) cells and PAF-R-deficient mice to demonstrate that staphylococcal lipoteichoic acid (LTA), a constituent of Gram-positive bacteria cell walls, acts as a PAF-R agonist. We show that LTA stimulates an immediate intracellular $\mathrm{Ca}^{2+}$ flux only in PAF-R-positive cells. Intradermal injections of LTA and the PAF-R agonist 1-hexadecyl-2- $N$-methylcarbamoyl glycerophosphocholine (CPAF) induced cutaneous inflammation in wild-type but not PAF-R-deficient mice. Systemic exposure to LTA or CPAF inhibited delayed-type hypersensitivity (DTH) reactions to the chemical dinitrofluorobenzene only in PAF-R-expressing mice. The inhibition of DTH reactions was abrogated by the addition of neutralizing antibodies to IL-10. Finally, we measured levels of LTA that were adequate to stimulate PAF-R in vitro on the skin of subjects with infected atopic dermatitis. Based on these studies, we propose that LTA exerts immunomodulatory effects via the PAF-R through production of the Th2 cytokine IL-10. These findings show a novel mechanism by which staphylococcal infections can inhibit Th1 reactions and thus worsen Th2 skin diseases, such as atopic dermatitis.
\end{abstract}

\section{Introduction}

Bacterial skin infections with Staphylococcus aureus cause significant morbidity and can even result in mortality. Skin infection or even colonization with $S$. aureus is a known trigger for inflammatory skin diseases, especially atopic dermatitis $(1,2)$. It should be noted that patients with atopic dermatitis are particularly susceptible to $S$. aureus infections, in part due to lack of endogenous antimicrobial peptides (3). The mechanisms by which staphylococcal infections can modulate immune responses are an active area of study. Through their ability to act as superantigens, staphylococcal exotoxins can activate large numbers of T cells and MHC class II-expressing cells (4-6). Lytic toxins, including $\alpha$ toxin, are potent stimulators of cytokine production at low doses but can induce cell death at higher levels (5-7).

Teichoic acid and peptidoglycan (PDG) are major polysaccharides in Gram-positive cell walls. Teichoic acid is also presently linked to a lipid moiety as lipoteichoic acid (LTA), which, like PDG, can signal through the TLR2 $(8,9)$. It should be noted that several studies have demonstrated that, unlike PDG, LTA can bind to the receptor for platelet-activating factor (PAF-R) (10-12). The ability of LTA to signal through the PAF-R is potentially clinically rel-

Nonstandard abbreviations used: CPAF, 1-hexadecyl-2- $N$-methylcarbamoyl glycerophosphocholine; DNFB, dinitrofluorobenzene; DTH, delayed-type hypersensitivity; GPC, glycerophosphocholine; LTA, lipoteichoic acid; PAF, platelet-activating factor; PAF-R, PAF receptor; PDG, peptidoglycan.

Conflict of interest: The authors have declared that no conflict of interest exists.

Citation for this article: J. Clin. Invest. 115:2855-2861 (2005).

doi:10.1172/JCI25429. evant in lung disease; Lemjabber and Basbaum have demonstrated that this bacterial cell wall constituent can augment mucous production in lung epithelial cells via the PAF-R (10).

PAF (1-O-alkyl-2-acetyl glycerophosphocholine) is a glycerophosphocholine-derived (GPC-derived) mediator with diverse functions (reviewed in ref. 13). Through its ability to both attract and activate leukocytes, this mediator has potent proinflammatory effects. PAF is produced in a wide variety of cell types; in keratinocytes its synthesis is stimulated by physical agents including ionophores, ultraviolet radiation, and heat/cold trauma (14-17). PAF exerts its effects via the PAF receptor, a $G$ protein-coupled receptor found on leukocytes as well as keratinocytes. Activation of the keratinocyte PAF-R results in the production of numerous lipid and protein cytokines, including IL-1, IL-6, IL-8, IL-10, TNF- $\alpha$, COX-2, prostanoids, and PAF itself (18-21). Recent studies have provided evidence that PAF-R agonists inhibit murine delayed-type hypersensitivity (DTH) reactions to Candida albicans (21). The present studies tested the hypothesis that LTA could exert immunomodulatory effects through its ability to act as a PAF-R agonist and assessed whether pharmacological amounts of LTA are found on lesions of infected atopic dermatitis. The current findings describe a putative mechanism by which staphylococcal bacteria can exert proinflammatory and immunomodulatory effects, which is relevant to the known ability of $S$. aureus to worsen skin diseases, especially atopic dermatitis.

\section{Results}

The KB PAF-R model system. Since PAF may have both receptordependent and -independent effects (secondary to the formation 

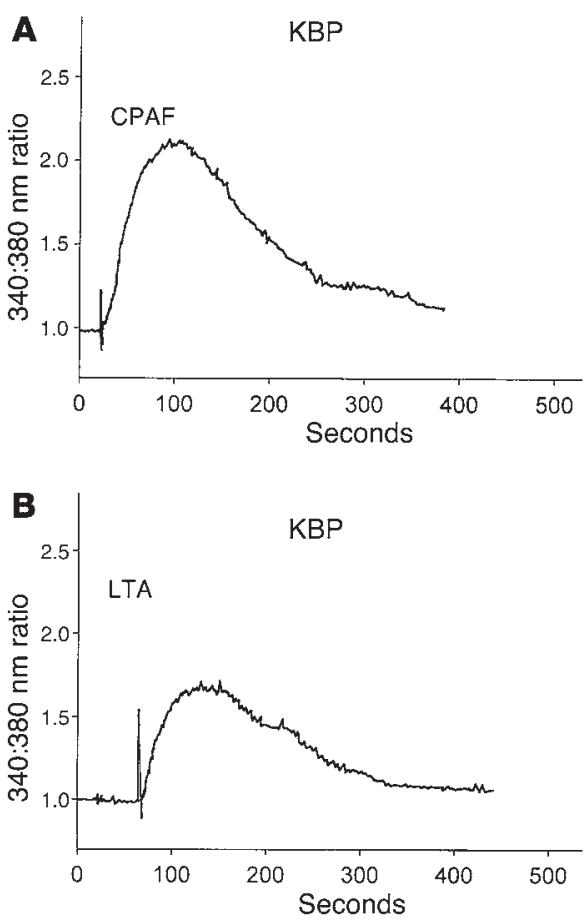

Figure 1

Calcium mobilization responses of KBP and KBM cells in response to CPAF and LTA. PAF-R-positive $\mathrm{KBP}(\mathbf{A}$ and $\mathbf{B})$ and PAF-R-negative $\mathrm{KBM}(\mathbf{C})$ cells were loaded with the $\mathrm{Ca}^{2+}$ sensitive dye Fura2 and treated with $100 \mathrm{nM}(54 \mathrm{ng} / \mathrm{ml})$ CPAF or $100 \mu \mathrm{g} / \mathrm{ml} \mathrm{LTA}$. Fluorescence intensity was measured over time with a spectrophotofluorimeter. Both CPAF and LTA stimulated immediate intracellular $\mathrm{Ca}^{2+}$ flux in KBP cells. LTA (C) and CPAF (not shown) had no effect on KBM cells, yet $100 \mathrm{nM}$ endothelin-1 (ET-1) stimulated an intracellular $\mathrm{Ca}^{2+}$ response in KBM cells. (D). KBP cells were treated with various concentrations of CPAF or LTA or $100 \mu \mathrm{g} / \mathrm{ml} \mathrm{PDG}$ and the peak change in intracellular $\mathrm{Ca}^{2+}$ determined. The data pictured are the mean \pm SEM from 3 separate experiments.

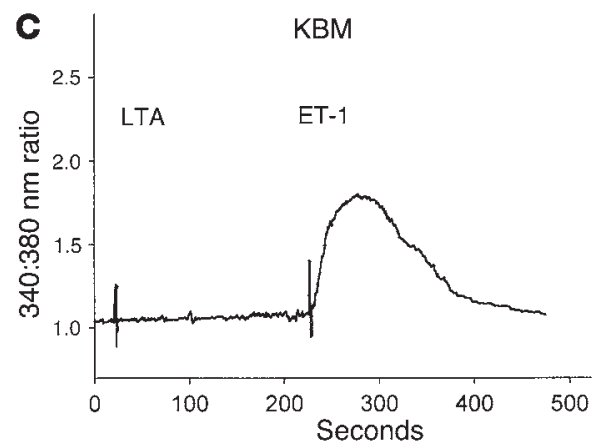

response in PAF-R-negative KBM cells. As a positive control, treatment of KBM cells with the G-protein coupled receptor ligand endothelin-1 resulted in an intracellular calcium mobilization (Figure 1C). LTA induced an intracellular $\mathrm{Ca}^{2+}$ flux in KBP cells in a dose-dependent manner, with concentrations greater than $500 \mathrm{ng} / \mathrm{ml}$ resulting in responses (Figure 1D). Treatment of KBP cells with the known TLR2 agonist PDG (23) at $100 \mu \mathrm{g} / \mathrm{ml}$ did not result in an intracellular $\mathrm{Ca}^{2+}$ flux (Figure 1D). These findings suggest that LTA can signal through the PAF-R but appears to be a weaker agonist than native PAF.

To determine whether LTA exerts its effects via direct activation of the PAF- $R$, radioligand binding studies were employed to assess whether this bacterial product could compete with $\left[{ }^{3} \mathrm{H}\right]$ WEB2086 for binding to KBP cells. Three separate experiments indicated that KBP cells specifically bound $323 \pm 24$ (mean \pm SEM) fmoles $\left[{ }^{3} \mathrm{H}\right]$ WEB2086/106 cells. Addition of $100 \mu \mathrm{g} / \mathrm{ml}$ LTA resulted in a decrease to $202 \pm 22$ fmoles of $\left[{ }^{3} \mathrm{H}\right]$ WEB2086/10 6 cells. In contrast, incubation with $100 \mu \mathrm{g} / \mathrm{ml}$ of the TLR2 agonist PDG resulted in similar amounts of specifically bound $\left[{ }^{3} \mathrm{H}\right]$ WEB2086 $(310 \pm 16$ fmoles $/ 10^{6}$ cells) as binding buffer. These studies confirm that LTA can indeed bind to the epidermal PAF-R.

Intradermal injection of LTA induces cutaof biologically active metabolites), our laboratory previously created a cellular model system by transduction of the PAF-R into a PAF-R-deficient epidermal cell line to study the role of the PAF-R in epithelial cell biology. Unlike normal human keratinocytes and the human keratinocyte-derived carcinoma cell line HaCaT (22), the human epidermal carcinoma cell line KB does not express functional PAF-R. A PAF-R-positive KB cell line, KBP, was created by transducing $\mathrm{KB}$ cells with a replication-deficient MSCV2.1 retrovirus containing the human PAF-R cDNA. KB cells were also transduced with the retroviral empty vector alone to establish a transduction control cell line, KBM. Expression of the PAF-R protein was verified by binding studies using radiolabeled PAF-R antagonist WEB2086 (18). Calcium mobilization studies demonstrated that the PAF-R in the KBP cell line was functionally active (18). Therefore, this in vitro epidermoid cell system consists of both PAF-R-negative (KBM) and -positive (KBP) cells.

Effects of LTA on $\mathrm{Ca}^{2+}$ signaling and cytokine production in $\mathrm{KB}$ cells. The first studies assessed the ability of LTA to signal through the PAF-R. As shown in Figure 1, treatment of KBP cells loaded with the $\mathrm{Ca}^{2+}$ sensitive dye Fura-2 AM with the PAF-R agonist 1-hexadecyl-2$\mathrm{N}$-methylcarbamoyl glycerophosphocholine (CPAF) resulted in a rapid intracellular $\mathrm{Ca}^{2+}$ flux. Similarly, treatment of KBP cells with LTA triggered an immediate $\mathrm{Ca}^{2+}$ flux (Figure 1B). However, neither CPAF (not shown) nor LTA (Figure 1C) induced a $\mathrm{Ca}^{2+}$ neous inflammation. Activation of the epidermal PAF-R induces the production of numerous proinflammatory cytokines (18-20) that could result in skin inflammation. To assess whether cutaneous exposure to LTA can result in inflammation through its ability to act as a PAF-R agonist, PAF- $\mathrm{R}^{-/-}$mice were used (24). To that end, the dorsal sides of ears of wild-type C57BL/6 (PAF-R expressing) or PAF- $\mathrm{R}^{-/}$mice were injected intradermally with CPAF, LTA, PDG, or histamine while the other ears were injected with BSA vehicle as control. After 2 hours, 5 -mm punch biopsy samples were taken from the ears, and inflammation was assessed by weighing the specimens. The 2 -hour period was chosen as this is the time of maximal inflammation (as measured by tissue weight) we have previously determined for intradermal injection of CPAF in rats (25). As shown in Figure 2, A and B, both wild-type and PAF-R-/mice were equally responsive to the positive control, intradermal injection of histamine. The intradermal injection of CPAF or LTA induced cutaneous inflammation in the ears of wild-type mice. In contrast, PDG injection did not result in the levels of ear inflammation seen in response to CPAF or LTA in wild-type mice. Consistent with involvement of the PAF-R in the inflammatory response of CPAF or LTA, neither of these agents exerted significant levels of inflammation in PAF- $\mathrm{R}^{-/}$mice. Histological examination of tissue from ear biopsies revealed significant edema with scattered neutrophils and a few eosinophils in the tissue from biopsies of 
A
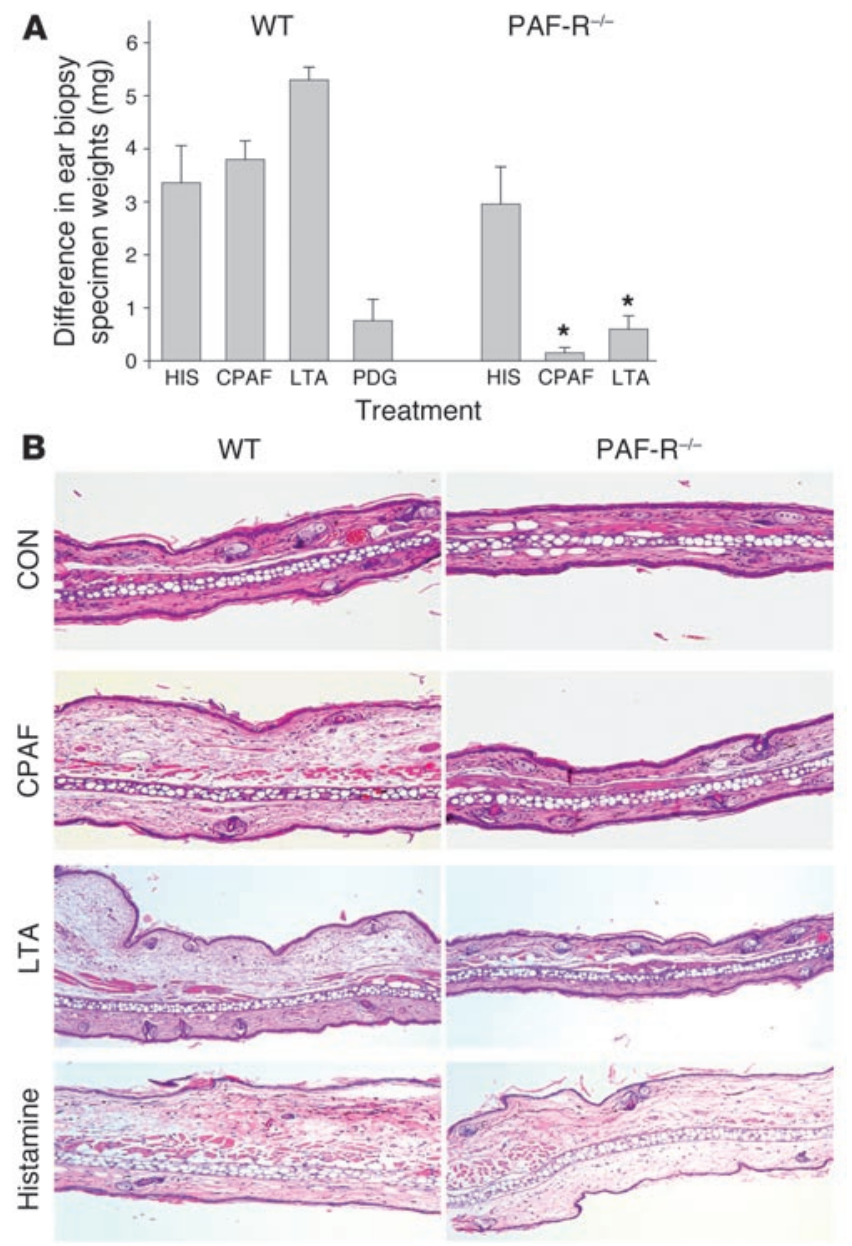

wild-type mice after intradermal injection of CPAF or LTA, compared with those in PAF- $\mathrm{R}^{-1-}$ mice (Figure 2B).

LTA suppresses DTH reactions. Systemic exposure to PAF results in an inhibition of murine DTH reactions to C. albicans, whose mechanism involves the COX-2-derived products (probably $\mathrm{PGE}_{2}$ ), as PAF-induced inhibition of this Th1 process was blocked by COX-2 inhibitors (21). It should be noted that a neutralizing antibody

\section{Figure 3}

Effects of CPAF and LTA on topical DTH reactions in wild-type versus PAF-R $\mathrm{R}^{-/-}$mice. (A) Effect of CPAF/LTA on DTH sensitization reactions. $250 \mathrm{ng}$ CPAF or $100 \mu \mathrm{g}$ LTA was injected i.p. into wild-type or PAF- $\mathrm{R}^{-/-}$mice. As described in Methods, the mice underwent sensitization to topical DNFB 5 days later, and 10 days later reactions were elicited by painting DNFB or vehicle on ears. Punch biopsies were performed 24 hours later, and the differences between biopsy specimen weights of DNFB- and vehicle-treated ears were measured using 6-8 mice in each group. (B) Effects of CPAF and LTA on DTH elicitation reactions. In these experiments, the mice were first sensitized to topical DNFB. Nine days after immunization, $250 \mathrm{ng}$ CPAF or $100 \mu \mathrm{g}$ LTA was injected i.p.; 1 day after injection, DTH reactions were elicited by painting DNFB or vehicle control on ears. Punch biopsies were performed 24 hours later, and the differences between biopsy specimen weights of DNFB- and vehicle-treated ears were measured using $7-8$ mice in each group. * ${ }^{*}$ tatistically significant $(P<0.05)$ decrease in difference of ear biopsy specimen weights of CPAF- or LTA-treated $\mathrm{PAF}^{-\mathrm{R}^{-/}}$mice in comparison with wild-type mice.

\section{Figure 2}

Effect of intradermal injections of CPAF, LTA, or PDG on skin inflammation. The dorsal sides of ears of wild-type and PAF- $\mathrm{R}^{-1-}$ mice were injected with $100 \mathrm{ng}$ CPAF, $10 \mu \mathrm{g}$ LTA, $10 \mu \mathrm{g}$ PDG (wild-type mice only), or $2.0 \mathrm{mg}$ histamine in 1 ear and BSA vehicle control on the other. After 2 hours, 5-mm punch biopsies were performed and tissues weighed, and the differences in ear biopsy specimen weights between CPAF/LTA versus BSA vehicle assessed. (A) Intradermal injection of CPAF or LTA induced local cutaneous inflammation (as measured by increased tissue weight) in wild-type but not PAF- $\mathrm{R}^{-1-}$ mice. The data consist of the mean \pm SD of the difference between LTA/PDG/CPAF/ histamine and vehicle-injected ear biopsy specimen weights using 6-7 mice in each group. *Statistically significant $(P<0.05)$ difference in ear thickness between WT and PAF- ${ }^{-1-}$ mice. (B) Representative H\&E-stained sections from CPAF-, LTA-, histamine-, or vehicle control-injected ear biopsy specimens revealed significant edema with scattered neutrophils and a few eosinophils in the skin of wild-type mice after intradermal injection of CPAF or LTA compared with essentially no effect on the ear skin of PAF- $\mathrm{R}^{-/-}$mice. Both wild-type and $\mathrm{PAF}-\mathrm{R}^{-/-}$mice were equally responsive to intradermal injection of histamine as positive control. CON, control. Magnification, $\times 100$.

against IL-10 can also abolish ultraviolet B radiation-mediated inhibition of murine DTH reactions $(26,27)$. The next studies were designed to assess whether LTA can inhibit DTH reactions via its ability to act as a PAF-R agonist. To investigate whether LTA can inhibit the sensitization of DTH, wild-type and PAF- $\mathrm{R}^{-/-}$mice were systemically exposed to either CPAF or LTA via intraperitoneal injection and 5 days later sensitized to the chemical compound dinitrofluorobenzene (DNFB). Nine days after DNFB sensitization, the dorsal sides of ears were treated with DNFB or vehicle control. Punch biopsy specimens were taken from ears 24 hours later and inflammation assessed by weighing and comparing DNFB- versus vehicle-painted biopsy tissues. As shown in Figure $3 \mathrm{~A}, \mathrm{CPAF}$ treatment inhibited subsequent sensitization reactions to DNFB in wild-type but not PAF-R $\mathrm{R}^{-/-}$mice. Similarly, exposure to
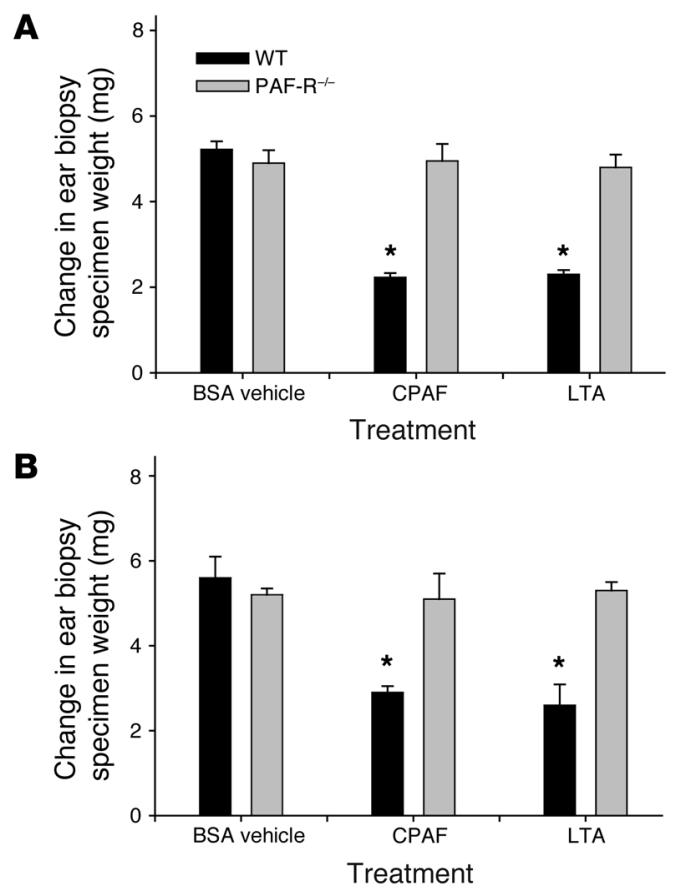

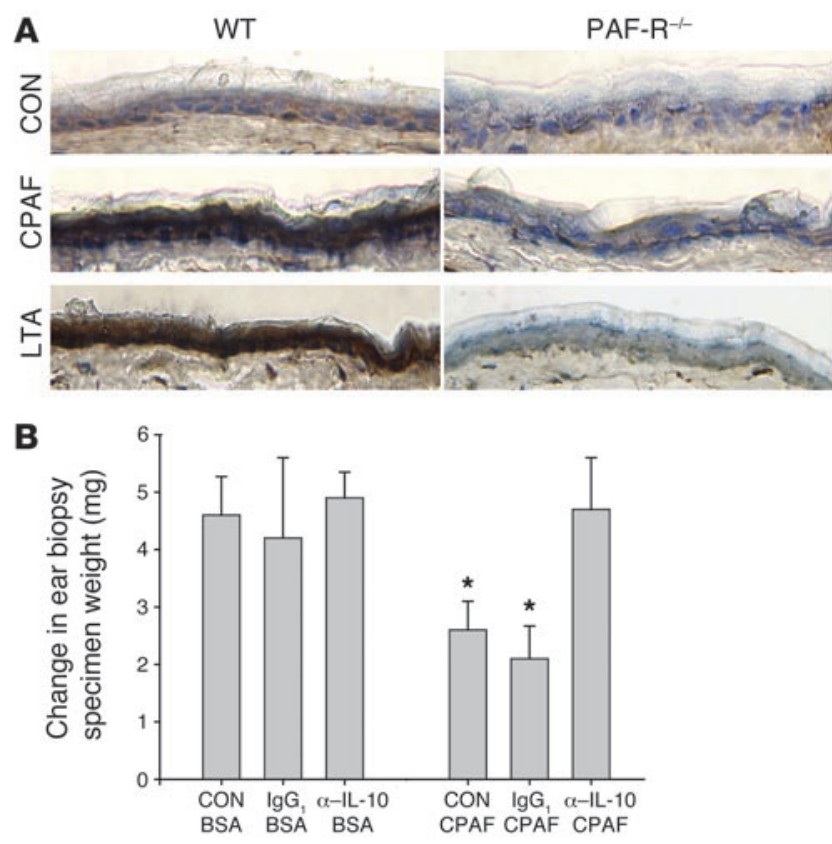

LTA inhibited this Th1 reaction only in mice expressing the PAF-R. To test whether CPAF or LTA could inhibit the elicitation reaction in mice sensitized to DNFB, both wild-type and PAF- $\mathrm{R}^{-/-}$mice were sensitized to DNFB and systemically exposed to CPAF or LTA 9 days after immunization. The day after CPAF/LTA treatment, the mice were challenged by DNFB ear painting as described above; ear biopsies were performed and tissues weighed 24 hours later. As shown in Figure 3B, both CPAF and LTA inhibited DTH elicitation reactions in wild-type but not PAF- $\mathrm{R}^{-/-}$mice.

Involvement of IL-10 in PAF-R-mediated suppression of DTH. Previous studies have shown that PAF-R activation can stimulate production of the Th2 cytokine IL-10, which is a known inhibitor for Th1 responses (21). The next studies were designed to examine the ability of CPAF or LTA to stimulate epidermal IL-10 production and the role of IL-10 in the PAF-R-mediated inhibition of DTH reactions. To that end, the dorsal sides of ears of wild-type and PAF- $\mathrm{R}^{-/}$mice were subjected to intradermal injection of CPAF, LTA, or vehicle control; the tissue was harvested at various times, and epidermal IL-10 was measured by immunohistochemistry. As shown in Figure 4A for the 72-hour time point, intradermal injection of CPAF induced epidermal IL-10 production in the ears of wild-type mice. Consistent with the previous report that PAF-R activation can induce IL-10 production in the murine keratinocyte cell line PAM 212 (21), the appearance of IL-10 in mouse epidermis was not apparent until 48 hours and was maximal around 72 hours after CPAF or LTA administration. Intradermal injections of CPAF did not result in the appearance of IL-10 in ear skin of PAF- $\mathrm{R}^{-/-}$mice. Of significance, LTA treatment also induced epidermal IL-10 production in wild-type but not PAF- $\mathrm{R}^{-/-}$mice in a manner similar to treatment with CPAF (Figure 4A).

To confirm that IL-10 is involved in PAF-R-mediated suppression of DTH reactions, we tested the ability of a neutralizing antibody against IL-10 (27) to block CPAF-induced inhibition of DNFB reactions. As shown in Figure 4B, systemic treatment with neutralizing anti-IL-10 antibody blocked the CPAF-induced inhibitory effects on DTH in wild-type mice. These studies provide

\section{Figure 4}

Involvement of IL-10 in PAF-R-mediated suppression of DTH. (A) Effect of CPAF or LTA injection on epidermal IL-10 levels. Dorsal sides of ears of wild-type or PAF- $\mathrm{R}^{-1-}$ mice were injected with $100 \mathrm{ng}$ CPAF, $10 \mu \mathrm{g}$ LTA, or vehicle control and the tissue harvested at various times. Immunohistochemistry was used to measure epidermal IL-10 levels. Depicted are representative sections 72 hours after treatment with CPAF, LTA, or vehicle control. Magnification, $\times 400$. (B) Effect of a neutralizing anti-IL-10 antibody on CPAF-mediated inhibition of DTH reactions. Wild-type mice were injected with $250 \mathrm{ng}$ CPAF or BSA vehicle control i.p.; 4 and 24 hours later, the mice were injected i.p. with $100 \mu \mathrm{g}$ neutralizing rat anti-mouse IL-10 or $100 \mu \mathrm{g}$ rat $\mathrm{IgG}_{1}$ or $\mathrm{BSA}$ vehicle. Five days later, the mice were sensitized with DNFB, then 9 days later challenged by painting DNFB on 1 ear and vehicle control on the other. Punch biopsies were performed and specimens weighed 24 hours later. The data represent the mean \pm SD difference in ear specimen weights from 7-8 mice. *Statistically significant $(P<0.05)$ inhibition of DNFBmediated DTH by CPAF treatment. It should be noted that treatment with a neutralizing anti-IL-10 antibody but not isotype $\operatorname{IgG}_{1}$ antibody control abolished CPAF-mediated inhibition of DTH reaction. the support for the notion that IL-10 is crucial for PAF-R-mediated inhibition of DTH reactions.

Measurement of LTA on infected dermatitis lesions. Given our findings that LTA could have significant proinflammatory and immunomodulatory effects and that staphylococcal infection is a known trigger for worsening of atopic dermatitis, the actual amounts of LTA associated with infected dermatitis lesions is relevant. Thus, the next studies assessed secondarily impetiginized dermatitis lesions for the presence of LTA by the method described by Williamson and Kligman (28). This method entails 2 sequential scrubbings of the surface of a defined area inside a sterile $2.5 \mathrm{~cm}$ diameter polypropylene tube using a mild detergent and a Teflon abrader. Wash specimens from 14 atopic dermatitis lesions (that appeared possibly secondarily impetiginized) derived from 9 subjects were tested for the presence of LTA by immunoblotting; S. aureus and Staphylococcus epidermidis bacteria were quantitated by limiting dilution assay. As shown in Figure 5 and Table 1, measurable levels of LTA were found in 9 of the 14 specimens. Statistical analysis showed that the levels of LTA positively correlated with the concentration of staphylococcal bacteria; the Spearman's Rank Correlation coefficient between LTA levels and concentration of all staphylococcal bacteria was $0.62(P=0.0174$, strong positive correlation). The coefficient between LTA levels and concentration of

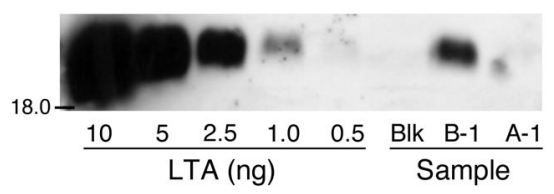

Figure 5

Examples of LTA measurement on samples from atopic dermatitis lesions. Standards of commercially available LTA (10, 5, 2.5, 1, 0.5, or $0[\mathrm{Blk}] \mathrm{ng}$ ) or wash solutions derived from clinically impetiginized lesions of atopic dermatitis (samples A-1 and B-1; see Table 1) were measured by immunoblotting as described in Methods. 


\section{Table 1}

LTA and staphylococcal levels in atopic dermatitis lesions

\begin{tabular}{lccc} 
Sample & $\begin{array}{c}\text { LTA } \\
\left(\mathbf{n g} / \mathbf{c m}^{3}\right)\end{array}$ & $\begin{array}{c}\text { S. aureus } \\
\left(\mathbf{c f u} / \mathbf{c m}^{2}\right)\end{array}$ & $\begin{array}{c}\text { S. epidermidis } \\
\text { (cfu/cm })\end{array}$ \\
A-1 & ND & 8 & $1.6 \times 10^{2}$ \\
B-1 & 641 & $1.2 \times 10^{5}$ & $6.2 \times 10^{6}$ \\
C-1 & ND & $1.6 \times 10$ & $4.0 \times 10^{2}$ \\
D-1 & ND & $1.2 \times 10^{5}$ & $1.2 \times 10^{5}$ \\
E-1 & 129 & $5.2 \times 10^{7}$ & 0 \\
E-2 & 712 & $5.1 \times 10^{6}$ & 0 \\
E-3 & 1355 & $2.0 \times 10^{7}$ & 0 \\
F-1 & 943 & $6.0 \times 10^{5}$ & 0 \\
F-2 & ND & $1.2 \times 10^{2}$ & $1.2 \times 10^{3}$ \\
G-1 & 161 & $4.0 \times 10^{6}$ & 0 \\
H-1 & 933 & $7.6 \times 10^{6}$ & $2.0 \times 10^{6}$ \\
H-2 & ND & $8.8 \times 10^{3}$ & 4 \\
I-1 & 1001 & $3.0 \times 10^{5}$ & 0 \\
I-2 & 739 & $2.5 \times 10^{6}$ & 0 \\
\hline
\end{tabular}

The amounts of LTA and S. aureus and coagulase-negative staphylococcus (S. epidermidis) bacteria were measured from a total of 14 atopic dermatitis lesions derived from $9(A-I)$ subjects as outlined in Methods. ND, not detected.

S. aureus was $0.66(P=0.0100$, strong positive correlation $)$, and the coefficient between LTA levels and concentration of coagulase-negative staphylococcus (S. epidermidis) was $-0.47(P=0.0877$, not significant). The amounts of LTA measured were expressed in Table 1 as $\mathrm{ng} / \mathrm{cm}^{3}$. This volume concentration was determined based upon the assumption that LTA would be on the surface of the epidermis at an approximate depth of $0.1 \mathrm{~cm}$. As shown in Figure 1D and Table 1, LTA concentrations adequate to activate the epidermal PAF-R ( $>500 \mathrm{ng} / \mathrm{ml}$ ) were found in 7 of the 14 samples. These studies indicate that significant amounts of LTA can be found on atopic dermatitis lesions when they become secondarily infected.

\section{Discussion}

Through the production of biologically active products, S. aureus can exert profound effects on skin inflammation. Characterization of the mechanisms by which $S$. aureus can modulate immune responses enhances our understanding of the role of bacterial infections in skin inflammation, especially in skin disease such as atopic dermatitis. These studies provide evidence for a novel mechanism for staphylococcal-mediated immune modulation, namely, that the bacterial cell wall product LTA can signal through the PAF-R.

Several lines of evidence suggest involvement of PAF-R in inflammatory skin diseases, especially atopic dermatitis. First, PAF has been measured in urticarial-like eruptions, including cold urticaria and bullous pemphigoid (29-31). Second, intradermal injection of PAF results in an urticarial wheal and flare reaction $(31,32)$. It should be noted that intradermal injection of PAF into atopic dermatitis patients resulted in enhanced reactions with increased numbers of eosinophils in comparison to nonatopic counterparts (32). Third, the lack of functional serum PAF acetylhydrolase, which catalyzes hydrolysis of PAF and short-chained sn-2 GPC, has been linked to a worse asthma phenotype in the approximately $4 \%$ of the Japanese population with homozygous mutations (33). Finally, the ability of PAF-R activation to stimulate production of the powerful Th2 cytokine IL-10 in epidermal and other cell types provides a mechanism by which the PAF system can inhibit Th1 and enhance Th2 responses (21). The importance of IL-10 in allergic skin diseases has been identified using tools such as IL-10-/mice, whose phenotype includes blunting of Th2 and enhanced Th1-associated reactions (34). The present studies demonstrating that PAF-R agonists can mimic the inhibitory effects of IL-10 on a Th1 model system of cutaneous DTH are consistent with the previous studies by Ullrich and colleagues indicating that PAF-R activation is an upstream signal for IL-10 production (21). Thus, activation of the PAF- $R$ could result in an immediate urticarial papule as well as later Th2 cytokine production, 2 features seen in worsening atopic dermatitis.

LTA-induced intracellular calcium mobilization was mediated through the PAF-R, as it was only seen in PAF-R-expressing KBP cells. It should be noted that LTA stimulated an immediate intracellular $\mathrm{Ca}^{2+}$ flux in KBP cells that mimicked the effect seen with the direct PAF-R agonist CPAF. This direct effect is in contrast to an agent such as staphylococcal $\alpha$-toxin that modulates intracellular $\mathrm{Ca}^{2+}$ signaling in PAF-R-expressing cells indirectly through the production of endogenous PAF, which has a lag of at least 1 minute before induction of the $\mathrm{Ca}^{2+}$ response (35). Our finding that LTA could displace $\left[{ }^{3} \mathrm{H}\right] \mathrm{WEB} 2086$ binding from KBP cells provides direct support for LTA acting directly on the epidermal PAF-R. LTA-induced PAF-R activation is probably not due to PAF contamination as select ion monitoring gas chromatography mass spectrometry evaluation using our previous published protocols (15-17) of commercial LTA samples (Sigma-Aldrich) did not reveal significant levels of PAF (data not shown). That the known TLR2 agonist PDG did not induce a calcium response or displace $\left[{ }^{3} \mathrm{H}\right]$ WEB2086-specific binding in KBP cells suggests that the LTA signaling effects did not involve TLR2. Based upon these findings and previous demonstrations that LTA and Gram-positive bacteria actually bind to the PAF-R $(10,12)$, it is proposed that LTA is acting as a direct agonist for the PAF-R.

The immunomodulatory effects of LTA in vivo were demonstrated by the finding that intradermal injection of LTA resulted in an almost immediate urticarial reaction containing neutrophils and scattered eosinophils. Moreover, systemic exposure to LTA suppressed both the sensitization and elicitation processes in a murine model of Th1 DTH reactions to the chemical DNFB. It should be noted that both the early cutaneous inflammatory response and the inhibitory effects on Th1 reactions were dependent upon the expression of PAF-R, as they were both absent in PAF-R $\mathrm{R}^{-/-}$mice.

The mechanism by which PAF-R activation could result in an inhibition of DTH responses was also explored. Since PAF-R activation has been shown to stimulate production of the potent Th2 cytokine IL-10 in the murine keratinocyte cell line PAM 212 (21), we examined whether IL-10 was involved in this process. Epidermal IL-10 production was shown to be positively induced by CPAF and LTA in wild-type but not PAF-R $\mathrm{R}^{-/-}$mice. In addition, the systemic administration of neutralizing anti-IL-10 antibody blocked CPAF-mediated inhibition of DTH in wild-type mice, providing direct evidence for the pivotal role of the Th2 cytokine IL-10 in the PAF-R-mediated suppression of DTH, a Th1 response.

The clinical significance of LTA-mediated inflammation and immune modulation via the PAF-R is unclear. However, our demonstration that significant levels of LTA are found on the skin from impetiginized atopic dermatitis lesions suggests that this bacterial product could be an important mediator of bacteriamediated worsening of eczema. Hence, the present studies provide one potential mechanism for the ability of staphylococcal skin 
infections to worsen Th2 skin diseases, such as atopic dermatitis. This pathway could be a pharmacological target through the use of PAF-R antagonists, including rupatadine, a novel antihistamine/PAF-R antagonist which has been shown to be effective for allergic rhinitis (36).

\section{Methods}

Reagents. All chemicals were obtained from Sigma-Aldrich unless otherwise indicated. PAF-R antagonist WEB2086 was a gift from Boehringer Ingelheim.

Cell culture. The human epidermoid cell line KB was grown in Dulbecco's modified Eagle's medium (Invitrogen Corp.) supplemented with $10 \%$ fetal bovine serum (HyClone). A KB PAF-R model system was created by transduction of PAF-R-negative KB cells with the MSCV2.1 retrovirus encoding the human leukocyte PAF-R as described previously (18). KB cells transduced with the PAF-R (KBP) or with control MSCV2.1 retrovirus $(\mathrm{KBM})$ were characterized by Southern and Northern blot analysis and by radioligand binding and calcium mobilization studies to demonstrate that the PAF-R was functional (18). All experiments were replicated with at least 2 separate $\mathrm{KBM}$ and $\mathrm{KBP}$ clones.

Radioligand binding studies. $\left[{ }^{3} \mathrm{H}\right] \mathrm{WEB} 2086$ binding studies were conducted as previously described (18). Briefly, KBP cells were plated into 24-well plates for 1 day and treated with $10 \mathrm{nM}\left[{ }^{3} \mathrm{H}\right]$ WEB2086 (New England Nuclear Corp.) with and without LTA, PDG, $1 \mu \mathrm{M}$ PAF, or binding buffer alone for 16 hours at $4^{\circ} \mathrm{C}$. Cells were washed and solubilized in Triton-X 100 and radioactivity counted using a scintillation counter (Beckman Coulter). Specifically bound $\left[{ }^{3} \mathrm{H}\right]$ WEB2086 was measured by the amount of radiolabel displaced by $1 \mu \mathrm{M}$ PAF. The data presented were mean \pm SEM from 3 separate studies using duplicate samples.

Calcium measurements. Intracellular $\mathrm{Ca}^{2+}$ mobilization studies in $\mathrm{KB}$ cells were conducted using Fura-2 AM (Invitrogen Corp.) as previously described (22).

Cutaneous inflammation measurements. PAF- $\mathrm{R}^{-/-}$mice on a C57BL/6 background were generated as previously described by Ishii and colleagues (24). Age-matched (8-12 weeks) PAF- $\mathrm{R}^{+/+} \mathrm{C} 57 \mathrm{BL} / 6$ wild-type mice were used as controls. All mice were housed in a pathogen-free environment, and studies were approved by the Animal Care and Use Committee of Indiana University School of Medicine. Intradermal injections of LTA, PDG, or CPAF were conducted as previously described with minor modifications (26). In brief, the dorsal sides of ears of anesthetized mice were injected with $50 \mu \mathrm{l} \mathrm{CPAF}(100 \mathrm{ng}), \mathrm{LTA}(10 \mu \mathrm{g})$, PDG $(10 \mu \mathrm{g})$, or histamine $(2.0 \mathrm{mg})$ in $0.25 \%$ fatty acid-free BSA and the other ears injected with $50 \mu \mathrm{lBSA}$ vehicle control. After 2 hours, 5 -mm punch biopsy specimens were taken from the ears and tissues weighed. After weighing, the tissues were placed in $10 \%$ buffered formalin, then paraffin embedded and stained with H\&E for routine histology.

IL-10 immunohistochemistry. The right ears of wild-type or PAF- $\mathrm{R}^{-/-}$mice were intradermally injected with $50 \mu \mathrm{l} \mathrm{CPAF}(100 \mathrm{ng})$ or LTA $(10 \mu \mathrm{g})$, and $0.25 \% \mathrm{BSA} / \mathrm{PBS}$ control was injected in left ears. The $6-\mathrm{mm}$ punch biopsy was performed at 24, 48, and 72 hours; the samples were then embedded in OCT, frozen in liquid nitrogen, and stored at $-80^{\circ} \mathrm{C}$. The goat $\mathrm{ABC}$ Staining System (Santa Cruz Biotechnology Inc.) was used according to the manufacturer's protocol. Briefly, $10-\mu \mathrm{m}$-thick cryostat sections of tissue block were cut and fixed in cold acetone for 10 minutes and washed in PBS 3 times for 5 minutes each time. The sections were then sequentially incubated in $1 \%$ hydrogen peroxide (in $\mathrm{H}_{2} \mathrm{O}$ ) for 10 minutes to block endogenous peroxidases, in $1.5 \%$ blocking serum in PBS for 60 minutes, then with primary antibody (1:100 dilution; Santa Cruz Biotechnology Inc.) overnight at $4{ }^{\circ} \mathrm{C}$. The slides were then incubated with biotinylated secondary antibody (1:150 dilution; Santa Cruz Biotechnology Inc.) with $\mathrm{AB}$ enzyme reagent for 30 minutes and incubated in 3 drops peroxidase substrate for 10 minutes or longer. Then sections were counterstained in Methyl Green (Vector Laboratories) at $60^{\circ} \mathrm{C}$ for 3 minutes and washed with $\mathrm{H}_{2} \mathrm{O}$ until clear. Slides were then dipped in acetone containing $0.05 \%(\mathrm{v} / \mathrm{v})$ acetic acid 10 times, immediately dehydrated, cleared, and permanently mounted.

DTH reactions. Studies of DTH reactions to 2, 4-DNFB were conducted as previously described (37) with minor modifications. In brief, to evaluate the effect of CPAF or LTA on sensitization reactions, both wild-type and PAF-R $-1-$ mice were injected i.p. with $50 \mu \mathrm{l} \mathrm{CPAF}(250 \mathrm{ng}), 50 \mu \mathrm{l} \mathrm{LTA}$ $(100 \mu \mathrm{g})$, or $50 \mu \mathrm{l}$ BSA vehicle alone. After 5 days, the back skin of each mouse was shaved, and $25 \mu \mathrm{l}$ of $0.5 \%$ DNFB in acetone/olive oil $(4: 1, v / v)$ was applied. Nine days later, 1 of the dorsal sides of the ear was challenged with painting of $10 \mu \mathrm{l}$ of $0.5 \% \mathrm{DNFB}$ and the other ear painted with vehicle. After 24 hours, 5-mm punch biopsy specimens were obtained from the ears and tissues weighed. For studies assessing the ability of CPAF or LTA to affect the elicitation phase of DTH reactions, both the wild-type and PAF- $\mathrm{R}^{-/-}$mice were first sensitized to DNFB. Nine days after immunization, the mice were injected i.p. with CPAF or LTA. The next day, the ears of mice were painted with DNFB or vehicle, and the ear biopsies were performed and tissues measured 24 hours after elicitation. To evaluate the effects of IL-10 in the CPAF-induced suppression of DTH, the mice were first injected i.p. with $250 \mathrm{ng}$ CPAF or BSA vehicle control; 4 and 24 hours later, the mice were twice injected i.p. with $100 \mu \mathrm{g}$ of neutralizing rat antimouse IL-10 antibody (BD Biosciences - Pharmingen) or $100 \mu \mathrm{g}$ rat $\mathrm{IgG}_{1}$ (eBioscience) as antibody control or BSA vehicle control; after 5 days, mice were sensitized with DNFB. Nine days later, the mice were challenged by painting of $10 \mu 10.5 \%$ DNFB on 1 ear and vehicle control on the other. The biopsies were performed 24 hours later and tissues weighed.

Measurement of LTA and bacteria in vivo. Nine subjects with atopic dermatitis diagnosed using criteria of Hanifen and Rajka (38) were enrolled in these studies, which were approved by the Indiana University Institutional Review Committee. Patients or their guardians gave informed consent for these studies. Wash fluid derived from 14 lesions was removed from a $2.5-\mathrm{cm}$ diameter polypropylene chamber using the methodology established by Williamson and Kligman (28). In brief, a sterile ring of PVC tubing (NALGENE Labware; Nalgene International) was placed over the skin lesion of a patient, then $1 \mathrm{ml}$ sterile rinse solution $\left(0.069 \mathrm{M} \mathrm{Na}_{2} \mathrm{HPO}_{4}, 0.0064 \mathrm{M}\right.$ $\mathrm{NaH}_{2} \mathrm{PO}_{4}$, and $0.1 \% \mathrm{Tx}-100$ ) was administered inside the ring chamber that was held tightly on the skin to prevent leakage. The rinse solution was stirred around in the chamber with a sterile Teflon rod (Scientific Commodities Inc.) for 15-20 times and collected. This collection was repeated twice, and $2 \mathrm{ml}$ total rinse solution was obtained. This methodology has been shown to be $95 \%$ quantitative for aerobic surface bacteria (28). The wash solution was then aliquotted for immunoblotting analysis of LTA protein and bacterial quantitation. For quantitative measurements of LTA protein, $32 \mu \mathrm{l}$ rinse solution from each patient sample was separated on $18 \%$ Tris- $\mathrm{HCl}$ gradient gel (Bio-Rad Laboratories) along with standards of 10, 5, 2.5 , and $1 \mathrm{ng}$ LTA protein (Sigma-Aldrich) dissolved in the same rinse solution loaded on the same electrophoresis gel. LTA protein was determined by immunoblotting with LTA monoclonal antibody (QED Bioscience Inc.) and enhanced chemiluminescence (Amersham Pharmacia Biotech). The arbitrary optical densities were measured by ImageJ Software (NIH; http:// rsb.info.nih.gov/ij/). The quantification of LTA was determined according to the standard curve drawn. LTA was quantitated based upon area $\left(\mathrm{ng} / \mathrm{cm}^{2}\right)$ that was then converted to volume $\left(\mathrm{ng} / \mathrm{cm}^{3}\right)$ based upon estimation of 0.1-cm effective epidermal thickness. S. aureus and S. epidermidis (coagulase-negative staphylococcus) colonies were quantitated in the microbiology lab in Indiana University Hospital by limiting dilution assay.

Data analysis. Data are presented as mean \pm SD of at least 3 independent experiments. Two-tailed Student's $t$ tests were used to assess statistical sig- 
nificance for differences in means, and Spearman's Rank Correlation coefficient was calculated to measure and test the strength of the association between levels of LTA and amounts of Gram-positive bacteria. Significance was set at $P<0.05$.

\section{Acknowledgments}

This research was supported in part by grants from the Showalter Memorial Foundation, the Riley Memorial Association, and the NIH (AR01993 and HL62996). The authors also wish to thank Dan Spandau and Raymond Konger for their critical reading of this manuscript. S. Ishii and T. Shimizu are supported by Grants-
in-Aid from the Ministry of Education, Culture, Sports, Science and Technology of Japan.

Received for publication April 21, 2005, and accepted in revised form July 12, 2005.

Address correspondence to: Jeffrey B. Travers, H.B. Wells Center for Pediatric Research, James Whitcomb Riley Hospital for Children, Room 2659, Indiana University School of Medicine, 702 Barnhill Drive, Indianapolis, Indiana 46202, USA. Phone: (317) 274-8804; Fax: (317) 274-5378; E-mail: jtravers@iupui.edu.
1. Leung, D.Y. 2003. Infection in atopic dermatitis. Curr. Opin. Pediatr. 15:399-404.

2. Spergel, J.M., and Paller, A.S. 2003. Atopic dermatitis and the atopic march. J. Allergy Clin. Immunol. 112:S128-S139.

3. Ong, P.Y., et al. 2002. Endogenous antimicrobial peptides and skin infections in atopic dermatitis. N. Engl. J. Med. 347:1151-1160.

4. McCormick, J.K., Yarwood, J.M., and Schlievert, P.M. 2001. Toxic shock syndrome and bacterial superantigens: an update [review]. Annu. Rev. Microbiol. 55:77-104.

5. Travers, J.B., Norris, D.A., and Leung, D.Y. 2001 The keratinocyte as a target for staphylococcal bacterial toxins. J. Investig. Dermatol. Symp. Proc. 6:225-230.

6. Ezepchuk, Y.V., et al. 1996. Staphylococcal toxins and protein A differentially induce cytotoxicity and release of tumor necrosis factor-alpha from human keratinocytes. J. Invest. Dermatol. 107:603-609.

7. Walev, I., et al. 1993. Staphylococcal alpha-toxin kills human keratinocytes by permeabilizing the plasma membrane for monovalent ions. Infect. Immun. 61:4972-4979.

8. Schwandner, R., Dziarski, R., Wesche, H., Rothe, M., and Kirschning, C.J. 1999. Peptidoglycan- and lipoteichoic acid-induced cell activation is mediated by toll-like receptor 2. J. Biol. Chem. 274:17406-17409.

9. Michelsen, K.S., et al. 2001. The role of toll-like receptors (TLRs) in bacteria-induced maturation of murine dendritic cells (DCS). Peptidoglycan and lipoteichoic acid are inducers of DC maturation and require TLR2. J. Biol. Chem. 276:25680-25686.

10. Lemjabbar, H., and Basbaum, C. 2002. Plateletactivating factor receptor and ADAM10 mediate responses to Staphylococcus aureus in epithelial cells. Nat. Med. 8:41-46.

11. Cundell, D.R., Gerard, N.P., Gerard, C., IdanpaanHeikkila, I., and Tuomanen, E.I. 1995. Streptococcus pneumoniae anchor to activated human cells by the receptor for platelet-activating factor. Nature. 377:435-438.

12. Garcia Rodriguez, C., et al. 1995. The role of N-glycosylation for functional expression of the human platelet-activating factor receptor. Glycosylation is required for efficient membrane trafficking. J. Biol. Chem. 270:25178-25184.

13. Prescott, S.M., Zimmerman, G.A., Stafforini, D.A., and McIntyre, T.M. 2000. Platelet-activating factor and related lipid mediators. Annu. Rev. Biochem.
69:419-445.

14. Michel, L., et al. 1990. Production of paf-acether by human epidermal cells. J. Invest. Dermatol. 95:576-581.

15. Travers, J.B., et al. 1996. Platelet-activating factor biosynthesis induced by various stimuli in human HaCaT keratinocytes. J. Invest. Dermatol. 107:88-94.

16. Travers, J.B. 1999. Oxidative stress can activate the epidermal platelet-activating factor receptor. J. Invest. Dermatol. 112:279-283.

17. Alappatt, C., Johnson, C.A., Clay, K.L., and Travers, J.B. 2000. Acute keratinocyte damage stimulates platelet-activating factor production. Arch. Dermatol. Res. 292:256-259.

18. Pei, Y., et al. 1998. Activation of the epidermal platelet-activating factor receptor results in cytokine and cyclooxygenase-2 biosynthesis. J. Immunol. 161:1954-1961.

19. Dy, L.C., Pei, Y., and Travers, J.B. 1999. Augmentation of ultraviolet B radiation-induced tumor necrosis factor production by the epidermal platelet-activating factor receptor. J. Biol. Chem. 274:26917-26921.

20. Southall, M.D., et al. 2001. The epidermal plateletactivating factor receptor protects epidermal cells from TNF- and TRAIL-induced apoptosis through a nuclear factor kappa B-dependent process. J. Biol. Chem. 276:45548-45554.

21. Walterscheid, J.P., Ullrich, S.E., and Nghiem, D.X. 2002. Platelet-activating factor, a molecular sensor for cellular damage, activates systemic immune suppression. J. Exp. Med. 195:171-179.

22. Travers, J.B., et al. 1995. Identification of functional platelet-activating factor receptors on human keratinocytes. J. Invest. Dermatol. 105:816-823.

23. Re, F., and Strominger, J.L. 2001. Toll-like receptor 2 (TLR2) and TLR4 differentially activate human dendritic cells. J. Biol. Chem. 276:37692-37699.

24. Ishii, S., et al. 1998 Impaired anaphylactic responses with intact sensitivity to endotoxin in mice lacking a platelet-activating factor receptor. J. Exp. Med. 187:1779-1788.

25. Travers, J.B., Pei, Y., Morin, S.M., and Hood, A.F. 1998. Antiinflammatory effects of the plateletactivating factor receptor antagonist A85783. Arch. Dermatol. Res. 290:569-573.

26. Shreedhar, V., Giese, T., Sung, V.W., and Ullrich, S.E. 1998. A cytokine cascade including prostaglandin E2, IL-4, and IL-10 is responsible for UVinduced systemic immune suppression. J. Immunol.
160:3783-3789.

27. Nghiem, D.X., et al. 2002. Mechanisms underlying the suppression of established immune responses by ultraviolet radiation. J. Invest. Dermatol. 119:600-608

28. Williamson, P., and Kligman, A.M. 1965. A new method for the quantitative investigation of cutaneous bacteria. J. Invest. Dermatol. 45:498-503.

29. Grandel, K.E., Farr, R.S., Wanderer, A.A., Eisenstadt, T.C., and Wasserman, S.I. 1985. Association of platelet-activating factor with primary acquired cold urticaria. N. Engl. J. Med. 313:405-409.

30. Okubo, K., Karashima, T., Hachisuk, H., and Sasai, T. 1989. The existence of a PAF-like substance in blister fluid derived from patients with bullous pemphigoid as demonstrated by human platelet aggregation. Kurume Med. J. 36:173-177.

31. Travers, J.B., et al. 1998. Identification and pharmacological characterization of platelet-activating factor and related 1-palmitoyl species in human inflammatory blistering diseases. Prostaglandins Other Lipid Mediat. 56:305-324.

32. Jenks, P.J., Kavanagh, G.M., Brooks, J., Bradfield, J.W., and Archer, C.B. 1999. Comparison of weal and flare responses to platelet activating factor $(\mathrm{PAF})$ and histamine, and the ultrastructural effects of PAF in the skin of atopic and normal subjects. Clin. Exp. Dermatol. 24:112-117.

33. Stafforini, D.M., et al. 1999. Deficiency of plateletactivating factor acetylhydrolase is a severity factor for asthma. J. Clin. Invest. 103:989-997.

34. Yang, X., Gartner, J., Zhu, L., Wang, S., and Brunham, R.C. 1999. IL-10 gene knockout mice show enhanced Th1-like protective immunity and absent granuloma formation following chlamydia trachomatis lung infection. J. Immunol. 162:1010-1017.

35. Travers, J.B., et al. 2003. Augmentation of staphylococcal alpha-toxin signaling by the epidermal platelet-activating factor receptor. J. Invest. Dermatol. 120:789-794.

36. Guadano, E.M., et al. 2004. Rupatadine $10 \mathrm{mg}$ and ebastine $10 \mathrm{mg}$ in seasonal allergic rhinitis: a comparison study. Allergy. 59:766-771.

37. Petersen, J.E., et al. 2002. Enhanced cutaneous inflammatory reactions to aspergillus fumigatus in a murine model of chronic granulomatous disease J. Invest. Dermatol. 118:424-429.

38. Hanifin, J.M., and Rajka, G. 1980. Diagnostic features of atopic dermatitis. Acta Derm. Venereol. (Stockh.). 92:44-47. 